\title{
Exhaled breath 8-isoprostane as a marker of asthma severity
}

Wojciech J. Piotrowski, Sebastian Majewski, Jerzy Marczak, Zofia Kurmanowska, Paweł Górski, Adam Antczak

Department of Pneumonology and Allergy, Medical University of Lodz, Poland

Submitted: 16 August 2010

Accepted: 12 January 2011

Arch Med Sci 2012; 8, 3: 515-520

DOI: $10.5114 /$ aoms.2012.28639

Copyright $\odot 2012$ Termedia \& Banach

\section{Abstract}

Introduction: Oxidative stress is a non-specific feature of airway inflammation in asthmatics. 8-Isoprostane (8-IP), a prostaglandin- $\mathrm{F}_{2 \alpha}$ isomer, is a relatively new marker of oxidative stress and may be measured in exhaled breath condensate $(E B C)$ of patients with asthma. This research study aimed to evaluate the usefulness of EBC 8-IP as a marker of severity and control of severe adult asthma.

Material and methods: Twenty-seven severe, never-smoking asthmatics were studied. According to positive or negative reversibility testing, this group was subdivided into reversible and irreversible asthma groups. All participants were observed for 8 weeks during which they completed daily diary observations including day and night symptoms, number of awakenings, peak expiratory flow (PEF) variability, daily rescue medication usage and oral steroids consumption. They attended the clinic 3 times and on these occasions spirometry assessments, EBC collection and asthma control tests (ACT) were done. Two control groups were included: 11 healthy never-smokers and 16 newly diagnosed and never-treated, non-smoking mild asthmatics.

Results: There were no statistically significant differences between severe asth$\mathrm{ma}$ and healthy control or never-treated asthma groups in concentrations of EBC 8-IP (median and interquartile range: $4.67 ; 2.50-27.92$ vs. $6.93 ; 2.5-12.98$ vs. $3.80 ; 2.50-10.73$, respectively). No correlations were found between EBC 8-IP and asthma control parameters, such as ACT results, night and day symptoms, consumption of rescue medication, percentage of days free of oral steroids, PEF diurnal variation, lung function test results, forced expiratory volume in the $1 \mathrm{~s}$ reversibility, and markers of systemic inflammation.

Conclusions: Our study results suggest that EBC 8-IP measurements are not useful for asthma monitoring.

Key words: 8-isoprostane, exhaled breath condensate, oxidative stress, severe asthma.

\section{Introduction}

Bronchial asthma is an inflammatory disease characterized by reversible bronchial obstruction. Good or at least partial control of the disease may be achieved in the majority of patients who are treated according to the international guidelines [1]. However, in about $10 \%$ of patients, asthma remains uncontrolled, despite intensive pharmacological treatment and good patient compliance. In difficult-to-treat asthma, uncontrolled inflammation may lead to remodelling of the bronchial wall and irreversible nar-

\author{
Corresponding author: \\ Adam Antczak MD, PhD \\ Department of Pneumonology \\ and Allergy \\ Medical University of Lodz \\ 22 Kopcinskiego \\ 90-153 Lodz, Poland \\ Phone: +48426782129 \\ Fax: +48 426782129 \\ E-mail: adam.antczak1@wp.pl
}


rowing of the bronchial lumen [2]. Consequentially, this form of asthma is more similar to chronic obstructive pulmonary disease (COPD) [3].

Oxidative stress is a non-specific feature of the inflammatory process, including the type present in the airways of asthmatics. Increased production of reactive oxygen species (ROS) by eosinophils, neutrophils, macrophages and other inflammatory cells may cause bronchial hyperreactivity, a key feature of asthma [4, 5]. Measurement of different biomarkers in exhaled breath condensate (EBC) is a relatively new research method which has raised the hope of easy, non-invasive and reliable monitoring of inflammation in the airways. Increased levels of nitrosothiols [6], nitrotyrosine [7], and especially nitric oxide $[8,9]$ have been detected in exhaled breath condensate of asthmatic patients. 8-Isoprostane (8-IP), a prostaglandin- $\mathrm{F}_{2 \alpha}$ isomer, produced in vivo by free radical-catalyzed peroxidation of arachidonic acid, is a relatively new marker of oxidative stress [10]. This marker has been measured in EBC of adults and children suffering from asthma [11-13], showing potential usefulness in disease monitoring. Children with problematic asthma had higher EBC 8-IP concentrations compared to patients with well-controlled disease [14]. Also, in adult patients 8-IP EBC concentrations were higher in severe compared to mild and moderate disease [15].

Therefore, the present study was designed to evaluate the usefulness of EBC 8-IP as a marker of severity and control of severe adult asthma.

\section{Material and methods}

\section{Study population}

Twenty-five severe, never-smoking asthmatics (age: $51.7 \pm 9.6$ years, 17 women) were studied. These patients were allowed to take all medications necessary to achieve the best possible level of asthma control during the whole study period. All were receiving inhaled corticosteroids (ICS) in the minimal dose of $2000 \mu \mathrm{g}$ budesonide or equivalent, long-acting $\beta$-agonists (LABA) and leukotriene modifying agents for at least 4 weeks before the start of the study. Short-acting $\beta$-agonists (SABA) were used as a rescue medication. Patients were free to take oral glucocorticosteroids either as a regular treatment or as a step-up regimen (5 mg to $40 \mathrm{mg}$ of prednisolone or equivalent daily). Therefore, on the basis of treatment options, the patients were classified either as GINA treatment stage 3 or 4 . In addition, forced expiratory volume in the $1 \mathrm{~s}\left(\mathrm{FEV}_{1}\right)$ before short-acting $\beta$-agonist (SABA) was $\leq 60 \%$ of predicted in all spirometric examinations performed in the last 12 months. In order to exclude a primary COPD and confirm the diagnosis of asthma, only never-smokers were included. Besides the above, one of the following criteria should have been fulfilled: positive reversibility test or methacholine challenge in the past, disease onset before the age of 40 years, and atopy confirmed by positive skin-prick tests or antigen-specific lgE in the peripheral blood. All patients were free of severe asthma exacerbation or respiratory infection for at least the last 6 weeks. Aspirin sensitivity was an exclusion criterion.

There were two control groups: 11 healthy never-smokers constituted a healthy control group (age: $46.6 \pm 12.3$ years, 5 women) and 16 newly diagnosed and never-treated, non-smoking mild asthmatics constituted a never-treated asthma control group (age: $32.0 \pm 8.5$ years, 9 women). In these patients, asthma was confirmed by typical symptoms and positive reversibility testing with SABA or methacholine challenge.

The study was approved by the Ethics Committee of the Medical University of Lodz. All patients gave written informed consent.

\section{Spirometry}

A flow-volume loop was performed according to Polish Respiratory Society recommendations [16] on a computer-based spirometer (Jaeger, Germany). Forced expiratory volume in the $1 \mathrm{~s}$ and forced vital capacity (FVC) were provided as percent of predicted value. $F_{E V} / F V C$ from best achieved values was calculated and presented as a percentage. The reversibility test was done $20-30 \mathrm{~min}$ after $400 \mu \mathrm{g}$ of inhaled salbutamol (see study protocol below).

\section{Exhaled breath condensate collection}

Exhaled breath condensate was collected using a condensing device (Ecoscreen, Jaeger, Germany). Patients were asked to breathe out spontaneously through a mouthpiece equipped with a saliva trap for $10 \mathrm{~min}$. The respiratory rate ranged from 15 breaths/ $\mathrm{min}$ to 20 breaths/min. All subjects wore a noseclip and rinsed their mouths with distilled water just before and after the $7^{\text {th }}$ min of the condensing process, in order to reduce saliva and nasal contamination. Samples were stored at $-80^{\circ} \mathrm{C}$ until measurement. The collection of EBC was performed following available recommendations [17].

\section{Measurement of 8-isoprostane}

Concentrations of 8-IP in EBC were measured by a specific enzyme immunoassay (EIA) kit (Cayman Chemical, Ann Arbour, MI) as previously described [12]. The detection limit (DL) was $5 \mathrm{pg} / \mathrm{ml}$. Levels of measured mediator below DL were arbitrarily assumed to be half of the DL value. All samples were measured in duplicate. The intra-assay reproducibility (coefficient of variation - CV) calculated for all measurements was $13.2 \%$. 
Study plan

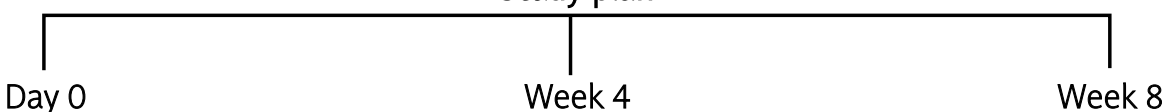

Spirometry + reversibility

EBC $\quad$ ACT
Spirometry \pm reversibility

Diary
Week 8

Spirometry \pm reversibility

ACT

PEF morning and evening

Oral steroids consumption

Rescue medication

No of awakenings

Symptoms (dyspnoea, cough, wheezing, impact on activity)

$E B C$ - exhaled breath condensate, $A C T$ - asthma control test

Figure 1. Study plan

\section{Other measurements}

Erythrocyte sedimentation rate (ESR) and C-reactive protein (CRP) as markers of systemic inflammation were measured. Patients' diaries included day and night symptoms, number of awakenings, PEF variability, daily rescue medication and oral steroids consumption. These features were reported throughout the 8 weeks. Asthma control tests (ACT) were performed at the beginning and at the end of the observation period. Asthma control tests consists of 5 questions evaluating the patient's activity, dyspnoea, night symptoms and awakenings, rescue medication consumption and selfassessment. The highest and lowest achievable result on the ACT was 25 and 5 points respectively [18].

\section{Study protocol}

Patients were under observation for 8 weeks. Spirometry with reversibility testing was performed three times in each patient (or less if reversibility was positive in $1^{\text {st }}$ or $2^{\text {nd }}$ examination) on different days (on the initial day, after 4 weeks and at the final visit), always in the morning and at least $6 \mathrm{~h}$ after the last intake of SABA, $8 \mathrm{~h}$ after ipratropium bromide and $24 \mathrm{~h}$ after the last dose of LABA or theophylline. Other drugs, such as inhaled or oral steroids and antileukotriene drugs, were allowed in the morning on the day of the spirometry assessments. The patients were assigned to the asthma reversible (A-RO) subgroup when, after $400 \mu \mathrm{g}$ of salbutamol, $\mathrm{FEV}_{1}$ increased more than $12 \%$ in relation to predicted and more than $200 \mathrm{ml}$ in relation to the pre-drug value in at least one test. The rest of the patients, who did not fulfil this condition, were assigned to the asthma irreversible obstruction subgroup (A-IRO). The study plan is presented in Figure 1.

\section{Statistical analysis}

Data are expressed as mean \pm standard error of means (SEM), with the exception of age, where standard deviation (SD) is provided. The Kolmogorov-Smirnov test was used to assess normality. A median with $25^{\text {th }}$ and $75^{\text {th }}$ percentile was provided for non-normally distributed data. One-way ANOVA and Bonferroni post-test (for data with Gaussian distribution) or Kruskal-Wallis followed by Dunn's multiple comparison test (for data without normal distribution) was used. Spearman test was

Table I. Patients' characteristics

\begin{tabular}{|c|c|c|c|}
\hline Parameter & All patients & Reversible obstruction & Irreversible obstruction \\
\hline Number of subjects & 25 & 9 & 16 \\
\hline Females & 16 & 5 & 11 \\
\hline Age [years] & $51.7 \pm 1.9$ & $49.7 \pm 2.3$ & $52.8 \pm 2.7$ \\
\hline Onset of asthma [years] & $31.9 \pm 2.9$ & $31.1 \pm 4.0$ & $32.4 \pm 4.0$ \\
\hline Duration of asthma [years] & $21.0 \pm 2.3$ & $19.7 \pm 3.4$ & $21.9 \pm 3.1$ \\
\hline $\mathrm{FEV}_{1}$ pre-dose [\% predicted] & $48.9 \pm 2.8$ & $50.7 \pm 5.2$ & $47.9 \pm 3.4$ \\
\hline $\mathrm{FEV}_{1} \% \mathrm{FVC}$ & $56.2 \pm 2.5$ & $60.0 \pm 4.5$ & $53.9 \pm 2.9$ \\
\hline Percent increase $\mathrm{FEV}_{1}$ post-dose & $9.7 \pm 1.2$ & $15.5 \pm 1.4$ & $6.2 \pm 0.9$ \\
\hline
\end{tabular}

$F E V_{1}$ - forced expiratory volume in the $1 \mathrm{~s}, F V C$-forced vital capacity 


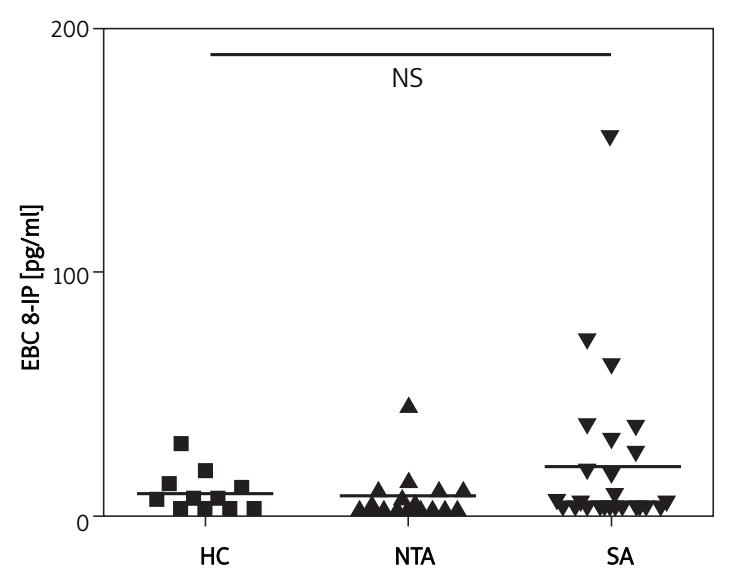

Figure 2. Concentrations of 8-isoprostane in exhaled breath condensate between study groups

$H C$ - healthy control group, NTA - never treated asthma group, SA - severe asthma group

applied to assess correlations. The $p$ value $\leq 0.05$ was assumed as statistically significant.

\section{Results}

The characteristics of the study group and results of pulmonary function tests (PFT) are presented in Table I. There were no statistically significant differences between severe asthma (SA) and healthy control (HC) or never-treated asthma (NTA) groups in concentrations of EBC 8-IP (median and interquartile range: $4.67 ; 2.50-27.92$ vs. $6.93 ; 2.5-12.98$ vs. $3.80 ; 2.50-10.73$, respectively) (Figure 2). Also, there was no statistically significant difference between patients with reversible (A-RO) and irreversible obstruction (A-IRO) within the SA group; however, it was noted that the A-IRO subgroup tended to have higher 8-IP concentrations (Figure 3). There was also no difference in EBC 8-IP between patients who were or were not on oral steroids at the time of EBC collection, nor between patients with uncontrolled (ACT $\leq 15, n=20$ ) and partly con-

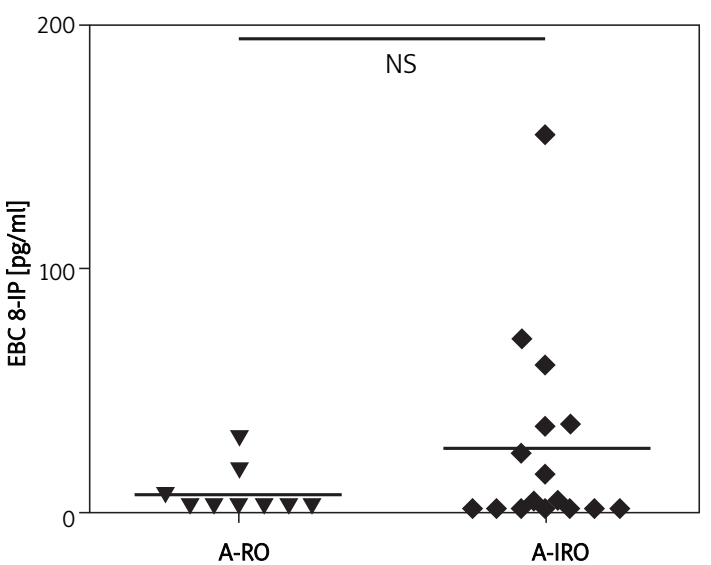

Figure 3. Concentrations of 8-isoprostane in exhaled breath condensate of severe asthma subgroups A-RO - asthma reversible obstruction subgroup, $A-I R O$ - asthma irreversible obstruction subgroup

trolled/controlled asthma (ACT $>15$, controlled $n=1$, partly controlled $n=4$ ) (data not shown).

No correlations were found between EBC 8-IP and asthma control parameters, such as ACT results, night and day symptoms, consumption of rescue medication, percentage of days free of oral steroids, PEF diurnal variation, PFT results, $\mathrm{FEV}_{1}$ reversibility, and markers of systemic inflammation. These parameters are shown in relation to asthma reversibility in Table II.

\section{Discussion}

With our present results, we could not confirm that EBC eicosanoids are elevated in asthma, as reported by other authors [11]. We can, however, confirm that there was a trend towards higher EBC 8-IP concentrations in severe asthma, both in comparison with healthy controls and mild never-treated asthmatics. Although the percentage of results below the detection limit was similar in all groups (36\%, 50\%, 48\% for HC, NTA and SA), the percent-

Table II. Comparison of severe asthma subgroups

\begin{tabular}{|lccc|}
\hline Feature & A-RO & A-IRO & Value of $p$ \\
\hline ACT [points] & $12.4 \pm 1.7$ & $13.2 \pm 1.4$ & NS \\
\hline Oral steroid-free days [\%] & $0(0-0)$ & $100(0-100)$ & - \\
& $0 \pm 0$ & $57 \pm 13$ & \\
\hline Symptom-free days [\%] & $0(0-0)$ & $0(0-0)$ & - \\
\hline Days with diurnal PEF variability $>$ 20\% [\%] & $6 \pm 4$ & $19 \pm 8$ & NS \\
\hline ESR [mm/h] & $6.8 \pm 1.6$ & $10.2 \pm 2.5$ & NS \\
\hline CRP & $2.2 \pm 0.5$ & $6.3 \pm 2.6$ & NS \\
\hline IgE total & $255.5 \pm 121.7$ & $145.2 \pm 48.1$ & NS \\
\hline Patients with positive specific lgE [\%] & 83 & 58 & NS \\
\hline
\end{tabular}

$A-R O$ - asthma reversible obstruction subgroup, A-IRO - asthma irreversible obstruction subgroup, ACT - asthma control test, $A C T$ - asthma control tests, ESR - erythrocyte sedimentation rate, CRP-C-reactive protein, IgE - immunoglobulin $E$ 
age of results above $20 \mathrm{pg} / \mathrm{ml}$ was $0 \%, 6 \%$ and $28 \%$, respectively. Also, the 8 -IP value did not differentiate severe asthmatics with reversible and irreversible obstruction. It should be mentioned here that results of 8-IP in EBC of asthmatics are difficult to compare between various laboratories [11-15, 19-23]. The differences may be attributed to technical aspects, such as the way of EBC collection, equipment used, storage conditions, etc. It is an important limitation of the method; therefore further studies should be addressed to standardization issues [17].

We did not find any correlations between 8-IP concentrations and features characterizing asthma control, such as ACT results and its components, percentage of symptom-free days or percentage of oral steroid-free days.

The loss of reversibility of bronchial obstruction is believed to be a typical physiopathological feature of airway remodelling in severe asthma. Therefore, we divided our group of severe asthmatics into those with reversible and irreversible bronchial obstruction. However, we did not find significant differences between these subgroups in terms of ACT and symptoms. The only striking difference is the lack of oral-steroid free days in the subgroup of asthmatics with reversible obstruction (100\% of patients and $100 \%$ of days on oral steroids), whereas 9 out of 16 patients with irreversible obstruction reported $100 \%$ of days free of oral steroids during the observation period. This may explain the trend of higher levels of 8-IP in the irreversible asthma subgroup. In order to substantiate this hypothesis, we included the analysis of a group consisting of mild newly diagnosed and never-treated asthmatics. But EBC 8-IP concentrations in this group were even lower, reflecting the minimal intensity of airway inflammation in these subjects. In agreement with this observation, Brussino et al. [19] did not find any differences between EBC 8-IP of episodic asthma and healthy controls.

We believe that one possible explanation for the lack of significant differences between our results and those of others in 8-IP concentration between controls and severe asthma is the influence of treatment. So far, several authors have reported the decrease of eicosanoid concentrations in EBC as a result of anti-inflammatory treatment in asthmatic patients. Although leukotriene $\mathrm{B}_{4}\left(\mathrm{LTB}_{4}\right)$ and 8 -IP seem to be resistant to inhaled steroids $[12,13$, 20], the influence of oral steroids or combination treatment may not be excluded $[13,21]$. However, the literature data in this matter are inconsistent. The reduction of 8-IP concentrations in EBC have been reported both in asthmatic and non-asthmatic patients with seasonal allergic rhinitis in response to treatment with nasal steroids [22]. Finally, due to the fact that inhaled steroids doses used in the cited papers were usually low to moderate, the possibility of the influence of high doses may not be excluded. In order to exclude such a possibility, a systematic study with a whole spectrum of doses of inhaled steroids should be performed in a homogeneous group of asthmatic patients. So far, these data are not available in the literature.

Only a few papers can be found reporting negative results. Kiełbasa et al. reported increased concentrations of cys-LT in EBC of 48 asthmatic children compared to healthy controls but 8-IP was not different [23].

Although our results do not neglect the possibility of increased concentrations of eicosanoids in asthma in general, the fact that they are not increased in severe and symptomatic asthma disqualifies the measurements of EBC 8-IP for asthma monitoring.

\section{Acknowledgments}

Supported by Medical University of Lodz, individual grant № 502-11-451 (Dr WJ Piotrowski). Many thanks to David Fam for linguistic corrections.

\section{References}

1. Gina Report, Global Strategy for Asthma Management and Prevention. Updated 2008. www.ginasthma.org. Accessed May 5th 2010.

2. Jenkins HA, Cool C, Szefler SJ, et al. Histopathology of severe childhood asthma: a case series. Chest 2003; 124: 32-41.

3. Frey U, Suki B. Complexity of chronic asthma and chronic obstructive pulmonary disease: implications for risk assessment, and disease progression and control. Lancet 2008; 372: 1088-99.

4. Rahman I, Morrison D, Donaldson K, MacNee W. Systemic oxidative stress in asthma, COPD, and smokers. Am J Respir Crit Care Med 1996; 154: 1055-60.

5. Ciencewicki J, Trivedi S, Kleeberger SR. Oxidants and the pathogenesis of lung diseases. J Allergy Clin Immunol 2008; 122: 456-68.

6. Corradi M, Montuschi P, Donnelly LE, Pesci A, Kharitonov SA, Barnes PJ. Increased nitrosothiols in exhaled breath condensate in inflammatory airway diseases. Am J Respir Crit Care Med 2001; 163: 854-8.

7. Hanazawa T, Kharitonov SA, Barnes PJ. Increased nitrotyrosine in exhaled breath condensate of patients with asthma. Am J Respir Crit Care Med 2000; 162: 1273-6.

8. Nightingale JA, Rogers DF, Barnes PJ. Effect of inhaled ozone on exhaled nitric oxide, pulmonary function, and induced sputum in normal and asthmatic subjects. Thorax 1999; 54: 1061-9.

9. Formanek W, Inci D, Lauener RP, Wildhaber JH, Frey U, Hall GL. Elevated nitrite in breath condensates of children with respiratory disease. Eur Respir J 2002; 19: 487-91.

10. Morrow JD, Frei B, Longmire AW, et al. Increase in circulating products of lipid peroxidation (F2-isoprostanes) in smokers. Smoking as a cause of oxidative damage. N Engl J Med 1995; 332: 1198-203.

11. Montuschi P, Corradi M, Ciabattoni G, Nightingale J, Kharitonov SA, Barnes PJ. Increased 8-isoprostane, a mark- 
er of oxidative stress, in exhaled condensate of asthma patients. Am J Respir Crit Care Med 1999; 160: 216-20.

12. Antczak A, Montuschi P, Kharitonov S, Gorski P, Barnes PJ. Increased exhaled cysteinyl-leukotrienes and 8-isoprostane in aspirin-induced asthma. Am J Respir Crit Care Med 2002; 166: 301-6.

13. Montuschi P, Nightingale JA, Kharitonov SA, Barnes PJ. Ozone-induced increase in exhaled 8-isoprostane in healthy subjects is resistant to inhaled budesonide. Free Radic Biol Med 2002; 33: 1403-8.

14. Carraro S, Cogo PE, Isak I, et al. EIA and GC/MS analysis of 8-isoprostane in EBC of children with problematic asthma. Eur Respir J 2010; 35: 1364-9.

15. Samitas K, Chorianopoulos D, Vittorakis S, et al. Exhaled cysteinyl-leukotrienes and 8-isoprostane in patients with asthma and their relation to clinical severity. Respir Med 2009; 103: 750-6.

16. Boros P, Franczuk M, Wesołowski S. Polish Respiratory Society recommendations for spirometry assessments [Polish]. Pneumonol Alergol Pol 2006; suppl. 1.

17. Horvath I, Hunt J, Barnes PJ, et al; ATS/ERS Task Force on Exhaled Breath Condensate. Exhaled breath condensate: methodological recommendations and unresolved questions. Eur Respir J 2005; 26: 523-48.

18. Nathan RA, Sorkness CA, Kosinski M, et al. Development of the asthma control test: a survey for assessing asthma control. J Allergy Clin Immunol 2004; 113: 59-65.

19. Brussino L, Badiu I, Sciascia S, et al. Oxidative stress and airway inflammation after allergen challenge evaluated by exhaled breath condensate analysis. Clin Exp Allergy 2010; 40: 1642-7.

20. Lehtimäki L, Kankaanranta $H$, Saarelainen S, et al. Bronchial nitric oxide is related to symptom relief during fluticasone treatment in COPD. Eur Respir J 2010; 35: 72-8.

21. Baraldi E, Carraro S, Alinovi R, et al. Cysteinyl leukotrienes and 8-isoprostane in exhaled breath condensate of children with asthma exacerbations. Thorax 2003; 58: 505-9.

22. Gratziou C, Rovina N, Makris M, Simoes DC, Papapetropoulos A, Roussos C. Breath markers of oxidative stress and airway inflammation in Seasonal Allergic Rhinitis. Int J Immunopathol Pharmacol 2008; 21: 949-57.

23. Kietbasa B, Moeller A, Sanak M, et al. Eicosanoids in exhaled breath condensates in the assessment of childhood asthma. Pediatr Allergy Immunol 2008; 19: 660-9. 